FTPI-MINN-06/19

June 26, 2006

UMN-TH-2506/06

hep-lat/0606003

\title{
Power-counting theorem for staggered fermions
}

\author{
Joel Giedt* \\ Fine Theoretical Physics Institute, University of Minnesota \\ 116 Church St. S.E., Minneapolis, MN 55455 USA
}

\begin{abstract}
Lattice power-counting is extended to QCD with staggered fermions. As preparation, the difficulties encountered by Reisz's original formulation of the lattice power-counting theorem are illustrated. One of the assumptions that is used in his proof does not hold for staggered fermions, as was pointed out long ago by Lüscher. Finally, I generalize the power-counting theorem, and the methods of Reisz's proof, such that the difficulties posed by staggered fermions are overcome.
\end{abstract}

*giedt@physics.umn.edu 


\section{Motivation and summary}

Lattice QCD with improved staggered fermions (SFs), or, Kogut-Susskind fermions [1-3], has recently enjoyed publicity for its ability to correctly reproduce many aspects of hadronic physics with reasonable accuracy [4,5]. However, SFs have some notable properties. For instance, SFs do not entirely overcome the fermion doubling problem. Rather, they reduce the number of continuum modes from 16 to 4 . (A further reduction to 2 modes is possible, by projecting quarks and antiquarks to odd and even sublattices resp. [3].) These 4 modes are referred to as tastes, to distinguish them from the $N_{f}$ flavors in the continuum theory. To estimate the fermion measure of $N_{f}$ continuum flavors, one takes the power $N_{f} / 4$ of the fermion determinant in the definition of the functional integral. I will not address the attendant controversy, but rather another technical question: lattice power-counting for staggered fermions. Here again, fermion doubling creates difficulties, as will be discussed at some length in this article, and as was pointed out some years ago by Lüscher [6].

To better understand perturbative renormalization of SFs it is of course useful to have a lattice power-counting theorem. By way of analogy, renormalizability of $S U(N)$ Yang-Mills coupled to Wilson fermions has been proven some time ago by Reisz [7]. This result was based on his earlier work on BPHZ-like renormalization theory on the lattice $[8,9]$. That work rested crucially on his lattice power-counting theorem $[10,11]$. (This literature is rather mathematical; more accessible reviews are those by Reisz $[12,13]$ and Lüscher [6].) Reisz's lattice power-counting theorem was a significant achievement because on the lattice Feynman integrands are trigonometric rather than rational functions of momenta; this can lead to results that differ from those of the continuum in important ways. ${ }^{1}$

It is often stated that no power-counting theorem exists for SFs; for example in Refs. [15-18]. However, it is also widely believed that the theory of SFs coupled to Yang-Mills (denoted here SF-QCD) yields the right quantum continuum limit in perturbation theory. That is to say, the lattice perturbation series can be renormalized and matched to a continuum renormalization scheme at every order in the gauge coupling $g$. This conclusion is supported by an analysis of the types of non-irrelevant operators that are allowed by the symmetries of SF-QCD. One finds that all such operators are already present at tree-level. (See for example [19] and refs. therein.)

\footnotetext{
${ }^{1}$ An amusing example occurs, for instance, in naive discretizations of supersymmetric quantum mechanics [14].
} 
That is, from a Wilsonian point of view one concludes that SF-QCD is in the same universality class as continuum QCD. It is reasonable to believe that by an adjustment of the bare parameters of the lattice action, one can arbitrarily adjust the coefficients of all non-irrelevant operators in the infrared, in order to obtain the desired theory.

The belief that SF-QCD is renormalizable also follows from a consideration of powers of the lattice spacing $a$ that arise in vertices and propagators of the theory, and how they appear in loop diagrams, an early example being [3]. In fact, for 1-loop diagrams, it is easy to power-count by partitioning the loop integration domain in a sensible way and estimating the integrand and measure for each of those domains. But this is nothing other than a limited version lattice power-counting. So, in fact, a version of power-counting already exists, though it is not as general as we would like. In actuality, this sort of partitioning is exactly what is done in Reisz's proof of his lattice power-counting theorem. However, the complexities that occur at high orders - where the number of domains increases factorially - are best addressed by a more sophisticated mathematical approach, just as in the continuum proofs of Weinberg [20] or Hahn and Zimmermann [21]. It is this sort of general method of power-counting that is aimed at in the present study.

I now summarize the remainder of this article:

- In $\oint_{2}$ I briefly review two well-known formulations of the SF-QCD action, and the corresponding Feynman rules.

- In 93 I review the conditions for the Reisz power-counting theorem. I also remind the reader of the lattice UV degree (of divergence) that is defined in Reisz's theorem.

- In 4 the conditions of the Reisz theorem are examined for the two formulations of SF-QCD thate were described in 92 . It is shown that in both cases the conditions of Reisz's theorem are violated. I explain the essential, basis-independent reason for this failure.

- In 95 I generalize Reisz's theorem and methods of proof in such a way that lattice power-counting can be applied to staggered fermions.

- In $₫ 6$ I conclude with a summary and discussion of further issues that could be explored.

Various appendices are included for details that would detract from the main discussion, but that are essential to the proof: 
- In $₫$ I discuss resolutions of identity that are used in a domain decomposition for the loop momenta integration. Both the one used by Reisz, and a generalized one that is applied in 95 are given.

- In $₫ \mathrm{~B}$ I discuss a simplification of the Feynman rules that is very useful in the momentum-space taste basis (MSTB).

- In $₫ \mathrm{C}$ it is shown that the domain of internal momenta can be extended in a useful way in the MSTB.

\section{Bases}

\subsection{The 1-component basis}

The gauge covariant $\mathrm{SF}$ action with link fields $U_{\mu}(r)$ is just [1-3]

$$
\begin{aligned}
S_{S F}= & \sum_{r \in \mathbf{Z}^{4}} \sum_{\mu=1}^{4} \frac{1}{2} a^{3} \alpha_{\mu}(r)\left[\bar{\chi}(r) U_{\mu}(r) \chi(r+\hat{\mu})-\bar{\chi}(r+\hat{\mu}) U_{\mu}^{\dagger}(r) \chi(r)\right] \\
& +\sum_{r \in \mathbf{Z}^{4}} m a^{4} \bar{\chi}(r) \chi(r), \quad \alpha_{\mu}(r) \equiv(-)^{r_{1}+\cdots+r_{\mu-1}} .
\end{aligned}
$$

Color indices are suppressed, $r$ is a site index, and $a$ is the lattice spacing. I refer to this as the 1-component basis (1CB). Under the lattice translation $\phi(r) \rightarrow \phi(r+s)$, with $\phi=U_{\mu}, \chi, \bar{\chi}$, the action is only invariant for even shifts $s \in 2 \mathbf{Z}^{4}$. Thus what I will call the Kähler-Dirac lattice ${ }^{2}$ consists of elementary cells that are hypercubes of length $b \equiv 2 a$ on each side, denoted $2 a \mathbf{Z}^{4}$.

The free fermion propagator $\left(U_{\mu} \equiv 1\right)$ has 16 lattice poles; i.e., minimal eigenvalues of the (Euclidean) SF Dirac operator. The 16-fold degeneracy corresponds to 4 continuum Dirac fermions. In momentum space, the additional poles lie at edges of the first Brillouin zone

$$
\mathcal{B}_{a}=(-\pi / a, \pi / a]^{4}
$$

More specifically, the poles lie at the sites of the lattice $(\pi / a) \mathbf{Z}^{4}$ that is reciprocal to the Kähler-Dirac lattice $2 a \mathbf{Z}^{4}$.

\footnotetext{
${ }^{2}$ This is the lattice generated by the basis vectors $2 \hat{\mu}$. I.e., those corresponding to translations that leave the lattice action invariant. It is the lattice through which free SFs are related to Kähler-Dirac fermions [22-26].
} 
Perturbation theory is, as usual, defined by expansion of

$$
U_{\mu}(r) \equiv \exp i a g A_{\mu}(r)
$$

in powers of $g$. Fourier transforms are defined with conventions:

$$
\phi(r)=\int_{\mathcal{B}_{a}} \frac{d^{4} k}{(2 \pi)^{4}} e^{i a r \cdot k} \tilde{\phi}(k), \quad \phi \in\left\{\chi, \bar{\chi}, A_{\mu}\right\},
$$

where $\mathcal{B}_{a}$ is defined in (2.2). I have chosen to make $k$ dimensionful, since it agrees with the conventions of Reisz. It will prove useful below to periodically extend the fields:

$$
\tilde{\phi}(k+(2 \pi / a) z) \equiv \tilde{\phi}(k), \quad \forall \quad z \in \mathbf{Z}^{4} .
$$

The form of $\tilde{A}_{\mu}(k)$ follows the convention of [19] but differs from the convention of $[27,28]$ by a factor of $e^{i a k_{\mu} / 2}$. The choice that is made here gives Feynman rules that are manifestly periodic on $\mathcal{B}_{a}$ since $\tilde{\phi}(k+(2 \pi / a) \hat{\nu})=\tilde{\phi}(k) \forall \nu \in\{1, \ldots, 4\}$, and $\tilde{\phi} \in\left\{\tilde{\chi}, \tilde{\bar{\chi}}, \tilde{A}_{\mu}\right\}$. In the conventions of $[27,28]$, slightly more effort must be expired to demonstrate periodicity of numerators of Feynman diagrams on $\mathcal{B}_{a}$, since individual vertex factors lack this property. $2 \pi / a$-periodicity of the numerator of Feynman integrands is an important assumption in Reisz's proof. See for example the Reisz-Lüscher conditions $\mathbf{V} \mathbf{1}$ and $\mathbf{C} \mathbf{1}$ in $\$ 3$,

For instance, in the conventions of $[27,28]$ the $\mathcal{O}(g)$ gluon-quark vertex is proportional to $\cos \left(p_{\mu} a+\frac{1}{2} k_{\mu} a\right)$, with $p$ incoming momentum on the $\tilde{\bar{\chi}}$ line and $k$ momentum on the incoming gluon line. Suppose $k$ is a loop momentum and we want to check the $2 \pi / a$-periodicity in condition $\mathbf{V} 1$. Under $k \rightarrow k+(2 \pi / a) \hat{\mu}$ the vertex reverses sign. This corresponds to $\tilde{A}_{\mu}(k+(\pi / a) \hat{\mu})=-\tilde{A}_{\mu}(k)$, due to the additional factor of $e^{i a k_{\mu} / 2}$ in the Fourier transform of $[27,28]$. However, one finds that the sign is always cancelled in some other part of the diagram that also involves the loop momentum $k$. This of course must be true, due to the equivalence with the formulation that I choose, where the Feynman rules themselves enjoy periodicity on $\mathcal{B}_{a}$.

The propagators and leading boson-fermion vertices are given, for instance, in Table 1 of [27], apart from the factor $e^{-i k_{\mu} a / 2}$ for each incoming $\tilde{A}_{\mu}(k)$. Let $\bar{\delta}_{a}$ denote the $2 \pi / a$-periodic $\delta$-function. Also define $\bar{\mu}=\sum_{\nu=1}^{\mu-1} \hat{\nu}$. Thus

$$
\overline{1}=(0,0,0,0), \quad \overline{2}=(1,0,0,0), \quad \overline{3}=(1,1,0,0), \quad \overline{4}=(1,1,1,0) .
$$

These are useful because in (2.1) we can write $\alpha_{\mu}(r)=\exp i \pi \bar{\mu} \cdot r$, which makes the Fourier transform easy to compute. 
Ignoring ghosts and pure YM vertices, ${ }^{3}$ the Feynman rules are:

$$
\begin{aligned}
D_{\mu \nu}^{c d}(k, \ell) & =\delta^{c d} \delta_{\mu \nu} \bar{\delta}_{a}(k+\ell)\left[\sum_{\mu} \frac{4}{a^{2}} \sin ^{2} \frac{k_{\mu} a}{2}+\lambda^{2}\right]^{-1}, \\
S^{i j}(p, q) & =\delta^{i j} \frac{m \bar{\delta}_{a}(p+q)-i a^{-1} \sum_{\mu} \sin \left(p_{\mu} a\right) \bar{\delta}_{a}\left(q+p+\frac{\pi}{a} \bar{\mu}\right)}{a^{-2} \sum_{\mu} \sin ^{2}\left(p_{\mu} a\right)+m^{2}}, \\
V_{\mu}^{c ; i j}(p, q ; k) & =-\frac{i}{2} g\left(T_{R}^{c}\right)^{i j}\left(e^{i p_{\mu} a}+e^{-i\left(p_{\mu}+k_{\mu}\right) a}\right) \bar{\delta}_{a}\left(k+p+q+\frac{\pi}{a} \bar{\mu}\right) .
\end{aligned}
$$

$T_{R}^{c}$ are generators of the gauge group in the quark representation $R ; i, j$ are color indices; $\lambda$ is an IR regulating mass for the gluon. $p, q$ are incoming momenta on the $\tilde{\bar{\chi}}, \tilde{\chi}$ lines respectively, whereas $k, \ell$ are incoming momenta on $\tilde{A}_{\mu}$ lines. Note that momentum conservation is only $\bmod \pi / a$ where the fermions are concerned (i.e., one finds $(\pi / a) \bar{\mu}$ inside the $\bar{\delta}_{a}$-functions), due to the fact that the Kähler-Dirac lattice is $2 a \mathbf{Z}^{4}$ (in physical units), which has for a reciprocal lattice $(\pi / a) \mathbf{Z}^{4}$.

\subsection{Momentum space taste basis}

Here I discuss the momentum space taste basis (MSTB) that was originally introduced in [3]. I present the results in conventions that are similar to [27, 28]; I retain the modification of the gluon Fourier transform that was discussed above. ${ }^{4}$

We make the following redefinition of the momentum space 1-component fields:

$$
\begin{aligned}
& \tilde{\chi}(k)=\chi_{A}\left(k_{r}\right), \quad \tilde{\bar{\chi}}(k)=\bar{\chi}_{A}\left(k_{r}\right), \quad k=k_{r}+\frac{\pi}{a} A \bmod \frac{2 \pi}{a}, \\
& k_{r}=\pi(k) \in \mathcal{B}_{2 a} \equiv(-\pi / 2 a, \pi / 2 a]^{4}, \\
& \left.A \in \mathcal{K}, \quad \mathcal{K} \equiv\left\{\left(0^{4}\right),\left(\underline{1,0^{3}}\right), \underline{1^{2}, 0^{2}}\right), \underline{\left(1^{3}, 0\right)},\left(1^{4}\right)\right\} .
\end{aligned}
$$

The notation is as follows. In the definition of the set of 4 -vectors $\mathcal{K}$, powers indicate how many times a 0 or 1 appears. Underlining indicates that all permutations of entries are to be included. Note that the 16 lattice poles described in 2.1 above are located in momentum space at $k \in(\pi / a) \mathcal{K}$. The map $\pi$ is a projection to the reduced

\footnotetext{
${ }^{3}$ There is no difficulty applying Reisz's power-counting theorem to pure YM. It is the SF propagator and vertices that pose problems. I include the gluon propagator (in Feynman gauge) for the purpose of illustrating how the YM sector is treated in tandem with SFs when PC is attempted. Treatment of ghosts is identical. A detailed analysis of pure YM interactions will not be required in what follows.

${ }^{4}$ There is also a position space taste basis [29], which I will not discuss here, except briefly in 4.3 For a more detailed exposition, consult also [30] as well as the reviews $[31,32]$.
} 
first Brillouin zone $\mathcal{B}_{2 a}$. Feynman vertices and propagators involving the fermions are then translated from the $1 \mathrm{CB}$ using this identification. In practice it is helpful to extend the definition as follows:

$$
\tilde{\chi}(k)=\chi_{A}\left(k^{\prime}\right), \quad \tilde{\bar{\chi}}(k)=\bar{\chi}_{A}\left(k^{\prime}\right), \quad k=k^{\prime}+\frac{\pi}{a} A, \quad \forall \quad k, k^{\prime} \in \mathbf{R}^{4}, A \in \mathcal{K} .
$$

Here, the periodically extended definitions [cf. (2.5)] of $\tilde{\chi}(k), \tilde{\bar{\chi}}(k)$ are used.

Taking these redefinitions into account, corresponding to (2.8) and (2.9) we have the Feynman rules [27,28]:

$$
\begin{aligned}
& S^{i j}\left(p=p^{\prime}+\frac{\pi}{a} A, q=q^{\prime}+\frac{\pi}{a} B\right) \equiv S_{A B}^{i j}\left(p^{\prime}, q^{\prime}\right) \\
& \quad=\delta^{i j} \bar{\delta}_{a}\left(p^{\prime}+q^{\prime}\right) \frac{m(\overline{\overline{1 \otimes 1}})_{A, B}-i a^{-1} \sum_{\mu} \sin \left(p_{\mu}^{\prime} a\right)\left(\overline{\overline{\gamma_{\mu} \otimes 1}}\right)_{A, B}}{a^{-2} \sum_{\mu} \sin ^{2}\left(p_{\mu}^{\prime} a\right)+m^{2}} \\
& V_{\mu}^{c ; i j}\left(p=p^{\prime}+\frac{\pi}{a} A, q=q^{\prime}+\frac{\pi}{a} B ; k\right) \equiv V_{\mu ; A B}^{c ; i j}\left(p^{\prime}, q^{\prime} ; k\right) \\
& =-\frac{i}{2} g\left(T_{R}^{c}\right)^{i j} \bar{\delta}_{a}\left(p^{\prime}+q^{\prime}+k\right)\left(e^{i p_{\mu}^{\prime} a}+e^{-i\left(p_{\mu}^{\prime}+k_{\mu}\right) a}\right)\left(\overline{\overline{\gamma_{\mu} \otimes 1}}\right)_{A, B} .
\end{aligned}
$$

As in (2.11), $p^{\prime}, q^{\prime}, k$ take any values in $\mathbf{R}^{4}$. An equivalence has been used to obtain momentum conserving $(\bmod 2 \pi / a) \bar{\delta}_{a}$-functions. It is reviewed in $\$ \mathrm{~B}$ and plays a crucial role in the generalized proof of $\$ 5$, The $16 \times 16$ momentum space spin-taste matrices $(\overline{\overline{1 \otimes 1}})_{A, B}$ and $\left(\overline{\overline{\gamma_{\mu} \otimes 1}}\right)_{A, B}$ are written in the notation of [27,28]. Definitions can be found therein; we will not need their explicit form in what follows.

The lattice perturbation theory also contains quark-multigluon vertices that are irrelevant operators, suppressed by explicit powers of the lattice spacing. They are important to take into account for the cancellation of divergences (see for example $\S 14.2$ of [31]), and are easily incorporated into the formalism that is discussed below. ${ }^{5}$

\section{Review of Reisz lattice power-counting}

\subsection{The lattice UV degree}

A given Feynman integral is written in the general form

$$
\hat{I}=\int_{\mathcal{B}_{a} L} d^{4} k_{1} \cdots d^{4} k_{L} \frac{V(k, q ; m, a)}{C(k, q ; m, a)} .
$$

\footnotetext{
${ }^{5}$ I thank David Adams for an important discussion regarding the role of the irrelevant vertices.
} 
Here, $k_{1}, \ldots, k_{L}$ are loop momenta and $q_{1}, \ldots, q_{E}$ are external momenta. Note that the loop momenta are integrated over $\mathcal{B}_{a}{ }^{L}=\times{ }^{L} \mathcal{B}_{a}=(-\pi / a, \pi / a]^{4 L}$; cf. (2.2). Also, $m$ stands collectively for mass parameters. The numerator $V$ incorporates vertex factors and the numerators of propagators; $C$ consists of a product of propagator denominators.

Reisz defines the UV degree of $V$ and $C$, and thence of the integral $\hat{I}$. At higher orders, this must be done over Zimmermann subspaces $H$. To each propagator corresponds a line momentum $\ell_{i}(k, q)$ (cf. (13.12) below). There is a sense in which $L$ of these form a basis w.r.t. $k_{1}, \ldots, k_{L}$, as will be explained in 3.3 below (cf. condition L2). We decompose this set as follows:

$$
\begin{array}{lll}
u_{1}=\ell_{i_{1}}, & \ldots, \quad u_{d}=\ell_{i_{d}} \\
v_{1}=\ell_{j_{1}}, & \ldots, & v_{L-d}=\ell_{j_{L-d}} .
\end{array}
$$

We regard $v_{1}, \ldots, v_{L-d}$ and $q_{1}, \ldots, q_{E}$ as external momenta to the Zimmermann subspace $H$. The $u_{1}, \ldots, u_{d}$ are regarded as internal momenta that provide a parameterization of $H$. We denote the set of all Zimmermann subspaces by $\mathcal{H}$.

The UV degree of $V$ w.r.t. $H$ is just the $\lambda \rightarrow \infty$ scaling exponent of $V$ as $u \rightarrow \lambda u$ and $a \rightarrow a / \lambda$. First we define:

$$
V(u, v, q ; m, a) \equiv V(k(u, v, q), q ; m, a)
$$

using the fact that the line momenta in (3.2) form a basis w.r.t. $k$. Then, as $\lambda \rightarrow \infty$ we extract the leading exponent: ${ }^{6}$

$$
V(\lambda u, v, q ; m, a / \lambda)=\lambda^{\nu} A(u, v, q ; m, a)+\mathcal{O}\left(\lambda^{\nu-1}\right) \quad \Leftrightarrow \quad \overline{\operatorname{degr}}_{\hat{u}} V=\nu .
$$

The UV degree of $C(k, q ; m, a)$ is defined similarly. Combining the two, we have

$$
\overline{\operatorname{degr}}_{\hat{H}} \hat{I}=4 d+\overline{\operatorname{degr}}_{\hat{u}} V-\overline{\operatorname{degr}}_{\hat{u}} C,
$$

where we recall that there are $d$ momenta internal to $H$.

\subsection{Reisz's theorem}

Convergence of the Feynman integral is then proven, provided

$$
\overline{\operatorname{degr}}_{\hat{H}} \hat{I}<0, \quad \forall \quad H \in \mathcal{H} .
$$

\footnotetext{
${ }^{6}$ Reisz adds a "hat" to the subscript of the degree operator in order to distinguish it from "the old polynomial degree."
} 
In this case, one obtains the remarkable result:

$$
\lim _{a \rightarrow 0} \hat{I}=\int_{-\infty}^{\infty} d^{4 L} k \frac{P(k, q ; m)}{E(k, q ; m)},
$$

where

$$
P(k, q ; m)=\lim _{a \rightarrow 0} V(k, q ; m, a), \quad E(k, q ; m)=\lim _{a \rightarrow 0} C(k, q ; m, a)
$$

are just the continuum limits of the numerator and denominator resp. Next I consider the conditions that are assumed to hold in the course of proving this result.

\subsection{The Reisz-Lüscher conditions}

In the proof of (3.5) and its consequences, Reisz makes some assumptions about the Feynman integrand. The lattice power-counting theorem of Reisz has been reviewed by Lüscher [6], and I will make use of his enumeration of the conditions that are assumed in the course of the proof. I refer to these as the Reisz-Lüscher conditions.

First, $V$ satisfies:

V1. There is an integer $\omega$ and function $F$ such that

$$
V(k, q ; m, a)=a^{-\omega} F(k a, q a ; m a)
$$

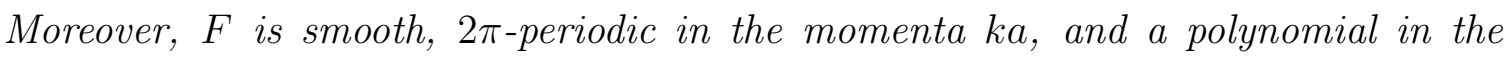
masses ma.

V2. $V$ has a continuum limit, in the sense that

$$
P(k, q ; m)=\lim _{a \rightarrow 0} V(k, q ; m, a)
$$

exists.

Lüscher notes that V1 - V2 are "not very restrictive." We will find that they are satisfied for SF-QCD.

As stated above, the denominator function $C$ that appears in (3.1) is a product of the denominators of propagators, $C_{1}, \ldots, C_{I}$ :

$$
C(k, q ; m, a)=\prod_{i=1}^{I} C_{i}\left(\ell_{i} ; m, a\right) .
$$

Here, each $C_{i}$ depends on a line momentum, described in more detail below. In $\mathrm{SF}-\mathrm{QCD}, C_{i}$ is a trigonometric function of the line momentum $\ell_{i}$. 
Reisz requires that the line momenta be natural, Defn. 3.1 in [10]. That is: ${ }^{7}$ L1*. The line momenta are of the form

$$
\ell_{i}(k, q)=\sum_{j=1}^{L} C_{i j} k_{j}+\sum_{\ell=1}^{E} D_{i \ell} q_{\ell} \equiv K_{i}(k)+Q_{i}(q) .
$$

Moreover, it is assumed that $C_{i j} \in \mathbf{Z}, D_{i \ell} \in \mathbf{R}$, and

$$
\operatorname{rank} C_{i j}=L, \quad\left(C_{i 1}, \ldots, C_{i L}\right) \neq 0 \quad \forall \quad i=1, \ldots, I \text {. }
$$

L2. Define the set

$$
\mathcal{L}=\left\{k_{1}, \ldots, k_{L}, K_{1}, \ldots, K_{I}\right\}
$$

where $K_{i}=\sum_{j} C_{i j} k_{j}$ were defined in (3.12). Suppose $u_{1}, \ldots, u_{L}$ are linearly independent elements contained in $\mathcal{L}$. Then the loop momenta can be expressed in terms of them with integer coefficients:

$$
k_{i}=\sum_{j=1}^{L} A_{i j} u_{j}, \quad A_{i j} \in \mathbf{Z} .
$$

Note that this property was used above in (3.3). It is in this sense that the line momenta appearing in (3.2) form a basis w.r.t. $k_{1}, \ldots, k_{L}$.

The following requirements are imposed on the functions $C_{i}$ that appear in (3.11): C1. The propagator denominators can be expressed as

$$
C_{i}\left(\ell_{i} ; m, a\right)=a^{-2} G_{i}\left(a \ell_{i} ; a m\right)
$$

where $G_{i}$ is a smooth function that is $2 \pi$-periodic in the momentum $\ell_{i} a$. Also, $G_{i}$ is a polynomial in the mass ma.

C2. The denominators have the conventional continuum limit:

$$
\lim _{a \rightarrow 0} C_{i}\left(\ell_{i} ; m, a\right)=\ell_{i}^{2}+m_{i}^{2}
$$

for fixed $\ell_{i}, m_{i}$. Here, $m_{i}^{2}$ is a homogeneous quadratic polynomial in the mass parameters of the theory.

\footnotetext{
${ }^{7}$ The asterisk denotes that I have modified Lüscher's statement of the condition in order to bring it into harmony with stricter definition given by Reisz.
} 
C3. There exists an $a_{0}>0$ and an $A>0$ such that

$$
\left|C_{i}\left(\ell_{i} ; m, a\right)\right| \geq A\left(\hat{\ell}_{i}^{2}+m_{i}^{2}\right), \quad \hat{\ell}_{i}^{2} \equiv \sum_{\mu} \frac{4}{a^{2}} \sin ^{2}\left(\ell_{i \mu} a / 2\right),
$$

for all $a \leq a_{0}$ and all $\ell_{i} \in \mathcal{B}_{a}$.

In Reisz's proof, he does not require $\mathbf{C} 3$, but instead a condition that has the same effect - a lower bound on the propagator denominator:

C3*. There exists an $a_{0}>0$ and an $A>0$ such that

$$
\left|C_{i}\left(\ell_{i} ; m, a\right)\right| \geq A\left(\ell_{i}^{2}+m_{i}^{2}\right),
$$

for all $a \leq a_{0}$ and all $\ell_{i} \in \mathcal{B}_{a}$.

Note that the r.h.s. of the inequality is a continuum expression. The reason that this is equivalent to $\mathbf{C} 3$ is that $C \equiv \hat{\ell}_{i}^{2}+m_{i}^{2}$ itself satisfies $\mathbf{C} 3^{*}$. Thus we can always replace the bound in $\mathbf{C} 3$ by the continuum (rational) expression in $\mathbf{C} 3 *$. In fact, the essence of Reisz's proof is to replace lattice expressions by bounds that are rational and have a continuum interpretation. For this reason I prefer C3*.

\section{The conditions of Reisz's theorem vs. staggered fermions}

Here I examine the Reisz-Lüscher conditions in relation to SFs. It will turn out that the Reisz-Lüscher conditions fail in both the $1 \mathrm{CB}$ and the MSTB. The essential reason is a mismatch between the Kähler-Dirac lattice $2 a \mathbf{Z}^{4}$ and the gauge lattice $a \mathbf{Z}^{4}$. (The former is the natural lattice on which to formulate free SFs, whereas the latter is the lattice on which the pure YM theory is formulated.)

In these considerations, it is implied that the $\delta$ functions that appear in the Feyman rules of $\S 2.1] 2.2$ have been integrated against (except for the overall $\delta$ function that always occurs), leading to the line momenta $\ell_{i}(k, q)$.

\subsection{1-component basis}

Consider the denominators of the propagators $D_{\mu \nu}^{c d}(k, \ell)$ and $S^{i j}(p, q)$ in relation to the Reisz-Lüscher conditions. For the gluon (B) and quark (F),

$$
C_{B}=a^{-2}\left[\sum_{\mu} 4 \sin ^{2} \frac{\ell_{\mu} a}{2}+(\lambda a)^{2}\right], \quad C_{F}=a^{-2}\left[\sum_{\mu} \sin ^{2}\left(\ell_{\mu} a\right)+(m a)^{2}\right],
$$


where $\ell$ is the line momentum flowing into the propagator. Both are of the form (3.16) and are periodic on $\mathcal{B}_{a}$; i.e., unchanged under $\ell \rightarrow \ell+(2 \pi / a)$. Thus $\mathbf{C 1}$ is satisfied. It is also obvious that $\mathbf{C 2}$ holds. Whereas $\mathbf{C} 3$ holds for $C_{B}$, it does not hold for $C_{F}$. This is because the latter has lattice poles away from the origin of $\mathcal{B}_{a}$, as I now show.

The proof consists of showing that there exist $\ell \in \mathcal{B}_{a}$ such that C3 fails. In particular, suppose that $\ell=(\pi / a) B$, where $B \in \mathcal{K}^{*} \equiv \mathcal{K}-\left(0^{4}\right)$. Then $C_{F}=m^{2}$ and $\hat{\ell}^{2}=4 a^{-2} \sum_{\mu} B_{\mu} \equiv 4 a^{-2}|B|$, where $\hat{\ell}^{2}$ was defined in (3.18). Let $A$ be any strictly positive real number. To satisfy $\mathbf{C} 3$ it is necessary that for sufficiently small $a_{0}$, and any $a<a_{0}$

$$
m^{2}-A\left(4 a^{-2}|B|+m^{2}\right) \geq 0
$$

This can only be true for $A<1$. But, for any a such that

$$
0 \leq a<a^{\prime} \equiv\left[\frac{4 A|B|}{(1-A) m^{2}}\right]^{1 / 2},
$$

condition (4.2) is violated. Thus we can never choose $a_{0}$ small enough to satisfy C3. Put simply, near one of the extra lattice poles, $C_{F}=\mathcal{O}\left(m^{2}\right)$ whereas $A\left(\hat{\ell}^{2}+m^{2}\right)=$ $A \mathcal{O}\left(a^{-2}\right)$; so, for small enough $a$ the latter is always larger.

The numerator of the quark propagator (2.8) will contribute to the Feynman numerator $V$ in (3.1), and it is easy to see that it satifies V1-V2. The $\bar{\delta}_{a}$-functions that appear are periodic on $\mathcal{B}_{a}$ by construction. It is always possible to choose loop momenta $k_{i}$ and line momenta $\ell_{i}$ such that $p \equiv \ell_{i}(k, q)$ in the denominator of (2.8), where $q$ is external momentum passing through the propagator. Due to the $\pi / a$ violations of momentum in the $1 \mathrm{CB}$, it is not guaranteed that the $\ell_{i}$ are natural; it is therefore possible that L1-L2 are also not satisfied. In any case $\ell_{i}=$ $C_{i j} k_{j}+Q_{i}(q)+z_{i}(\pi / a)$ with $C_{i j} \in \mathbf{Z}$ and $z_{i} \in \mathbf{Z}^{4}$. Then under $k_{j} \rightarrow k_{j}+(2 \pi / a) \hat{\nu}$, for any loop momentum $k_{j}$, the numerator term $\sum_{\mu} \sin \ell_{i \mu} a$ is unchanged. The vertex $V_{\mu}^{c ; i j}(k ; p, q)$ also satisfies $\mathbf{V} \mathbf{1}$, because it is has been constructed to be periodic on $\mathcal{B}_{a}$. It is obvious that the vertex satisfies $\mathbf{V} \mathbf{2}$.

Thus we see that the Reisz-Lüscher conditions fail to hold in the $1 \mathrm{CB}$ principally for the reason that has been pointed out by Lüscher [6]: the fermion propagator has too many poles in $\mathcal{B}_{a}$. Also worrisome is the $\pi / a$ violations of momentum. The latter problem will be eliminated in the basis that I discuss next. 


\subsection{Momentum space taste basis}

Note that the denominators of the MSTB propagators are the same as in the $1 \mathrm{CB}$. One has exactly the same violation of the Reisz-Lüscher conditions as in the $1 \mathrm{CB}$, due to additional quark poles in $\mathcal{B}_{a}$.

One might think to instead apply the Reisz-Lüscher conditions on the reduced Brillouin zone $\mathcal{B}_{2 a}$, defined in (2.10). This effectively replaces the lattice spacing $a$ by $b \equiv 2 a$. Whereas $\mathbf{C} 3$ is satisfied if $\mathcal{B}_{a}$ is replaced by $\mathcal{B}_{b} \equiv \mathcal{B}_{2 a}$, the Feynman rules do not enjoy $2 \pi / b=\pi / a$ periodicity. Thus $\mathbf{V} \mathbf{1}$ and $\mathbf{C} 1$ would be violated if we took this approach. In fact, in the generalization that is introduced in $\$ 5$ below, the lack of $\pi / a$-periodicity will be addressed "head-on".

\subsection{The mismatch}

The problem with $\pi / a$-periodicity is an inevitable consequence of the mismatch between the Kähler-Dirac lattice and the gauge lattice. To see this, note that the Fourier transform (2.4) has been formulated w.r.t. the translation invariance group of the gauge lattice, generated by shifts $\hat{\mu} a$. As a consequence, the fields $\tilde{\phi}(k)$ are periodic on the reciprocal lattice $(2 \pi / a) \mathbf{Z}^{4}$. If not for the gauge fields, we could perform a Fourier transform w.r.t. the translation invariance group of the Kähler-Dirac lattice, generated by shifts $\hat{\mu} b=2 \hat{\mu} a$. To accomplish this, we pass to the position space hypercube basis [28-30] before taking the Fourier transform:

$$
\begin{aligned}
& \chi(r=2 y+A) \equiv \chi_{A}(y), \quad y \in \mathbf{Z}^{4}, A \in \mathcal{K} ; \\
& \chi_{A}(y)=\int_{\mathcal{B}_{b}} \frac{d^{4} k}{(2 \pi)^{4}} e^{i b y \cdot k} \tilde{\chi}_{A}(k)=\int_{\mathcal{B}_{2 a}} \frac{d^{4} k}{(2 \pi)^{4}} e^{i 2 a y \cdot k} \tilde{\chi}_{A}(k) .
\end{aligned}
$$

It is easy to see that the quark propagator for the $\tilde{\chi}_{A}(k)$ fields is $\pi / a$-periodic. This just follows from the inverse Fourier transform:

$$
\tilde{\chi}_{A}(k)=b^{4} \sum_{y \in \mathbf{Z}^{4}} e^{-i b y \cdot k} \chi_{A}(y),
$$

which clearly leads to

$$
\tilde{\chi}_{A}(k+(\pi / a) z)=\tilde{\chi}_{A}(k) \quad \forall \quad z \in \mathbf{Z}^{4} .
$$

Thus the Feynman rules for $\tilde{\chi}_{A}(k+(\pi / a) z)$ and $\tilde{\chi}_{A}(k)$ will be identical. This is just to say that the reciprocal lattice of the Kähler-Dirac lattice is $(2 \pi / b) \mathbf{Z}^{4}=(\pi / a) \mathbf{Z}^{4}$. 


\section{The Reisz proof generalized}

I now extend the Reisz power-counting theorem and proof such that the deviations from the Reisz-Lüscher conditions can be overcome. The trick is to use:

(i) the MSTB rules with momentum-conserving $\delta$-functions,

(ii) integration extended to full periods,

(iii) resolution of identity on the reduced reciprocal lattice, and

(iv) tranformed Feynman rules that absorb loop momentum shifts

$$
k_{i} \rightarrow k_{i}+(\pi / a) A_{i}, \quad A_{i} \in \mathcal{K}
$$

The Feynman integral is written in the MSTB. The domain of integration is $\mathcal{B}_{a}$, using the trick of $₫ \mathrm{C}$. The vertices and propagators conserve momentum, using the equivalence of $₫ \mathrm{~B}$. Of course, one first integrates and sums against all $\delta$ functions. This should be done in such a way as to conserve momentum as it flows through the diagram, using the $2 \pi / a$-periodicity wherever necessary. ${ }^{8}$ Then we are left with just integrals over loop momenta, which can be routed such that the line momenta are natural. That is, the momenta are routed just as in a continuum Feynman integral.

\subsection{The denominator}

I now describe the modification that overcomes the principal difficulties posed by SFs. One breaks up the line momenta into those corresponding to bosons (gluons) and fermions (quarks): $\ell_{1}^{B}, \ldots, \ell_{I_{B}}^{B}$ and $\ell_{1}^{F}, \ldots, \ell_{I_{F}}^{F}$ resp. Then one inserts into the Feynman integral, for each $\ell_{i}$, the resolutions of identity that are described in $1_{B}\left(\ell_{i}^{B}\right)$ defined in (A.2) and $1_{F}\left(\ell_{j}^{F}\right)$ defined in (A.4). One obtains an expression analogous to Reisz's Eq. (4.4) [10] - a sum of integrals that comprises a domain decomposition:

$$
\begin{aligned}
& \hat{I}=\sum_{J_{B}, J_{F}} \sum_{z_{B}, z_{F}} \hat{I}\left(J_{B}, J_{F}, z_{B}, z_{F}\right) \equiv \sum_{J z} \hat{I}_{J z}, \\
& J_{B} \subseteq\left\{1, \ldots, I_{B}\right\}, \quad J_{F} \subseteq\left\{1, \ldots, I_{F}\right\}, \\
& z_{B}=\left(z_{B i} \mid i \in J_{B}\right), \quad z_{F}=\left(z_{F j} \mid j \in J_{F}\right),
\end{aligned}
$$

\footnotetext{
${ }^{8}$ This is optional because the MSTB Feynman rules only conserve momentum mod $2 \pi / a$.
} 
with individual terms of the form:

$$
\begin{aligned}
\hat{I}_{J z}= & \int_{\mathcal{B}_{a} L} \frac{d^{4 L} k V(k, q ; m, a)}{\prod_{i=1}^{I_{B}} C_{B}\left(\ell_{i}^{B} ; \lambda, a\right) \prod_{j=1}^{I_{F}} C_{F}\left(\ell_{j}^{F} ; m, a\right)} \\
& \times \prod_{i \in J_{B}} \Theta\left(\frac{\pi}{a} \epsilon-\left\|\ell_{i}^{B}-\frac{2 \pi}{a} z_{B i}\right\|\right) \prod_{i \notin J_{B}} \Theta_{\epsilon}^{B}\left(\ell_{i}^{B}\right) \\
& \times \prod_{j \in J_{F}} \Theta\left(\frac{\pi}{a} \epsilon-\left\|\ell_{j}^{F}-\frac{\pi}{a} z_{F j}\right\|\right) \prod_{j \notin J_{F}} \Theta_{\epsilon}^{F}\left(\ell_{j}^{F}\right) .
\end{aligned}
$$

Note that $J$ collectively denotes $J_{B}, J_{F}$, and so on. The decomposition has the following intuitive meaning: $\ell_{i} \in J$ are " $\epsilon$-near" to a lattice pole, whereas $\ell_{i} \notin J$ are " $\epsilon$-far" from a lattice pole.

For $\epsilon, a$ sufficiently small, the arguments of Reisz's Appendix D [10] extend in an obvious way to show that there exists $k^{(0)} \equiv\left(k_{1}^{(0)}, \ldots, k_{L}^{(0)}\right) \in \mathcal{B}_{a}{ }^{L}$ s.t.:

$$
K_{i}^{B}\left(k^{(0)}\right)=\frac{2 \pi}{a} z_{B i}, \quad K_{j}^{F}\left(k^{(0)}\right)=\frac{\pi}{a} z_{F j}, \quad i \in J_{B}, \quad j \in J_{F} .
$$

Note that $K_{i}(k)$ was defined in (3.12). Using the fact that $\ell_{i}$ are natural, it is a trivial extension of Reisz's Lemma D.2 [10] to prove that there exist reduced reciprocal lattice vectors

$$
\Delta_{1}, \ldots, \Delta_{L} \in \frac{\pi}{a} \mathbf{Z}^{4}
$$

such that for $i \in J_{B}, j \in J_{F}$

$$
K_{i}^{B}(\Delta)=\frac{2 \pi}{a} z_{B i}, \quad K_{j}^{F}(\Delta)=\frac{\pi}{a} z_{F j}
$$

The $\Delta_{i}$ are determined in terms of a basis chosen from $\left\{K_{i}^{B}, K_{j}^{F}\right\}$, as explained in Reisz's Appendix D [10]. Thus we define new loop momenta $k_{i}^{\prime}$ through:

$$
k_{i}=k_{i}^{\prime}+\Delta_{i} \equiv k_{i}^{\prime}+\frac{\pi}{a} \delta_{i} \quad \forall \quad i=1, \ldots, L,
$$

where in the last step integer-valued 4-vectors $\delta_{i}$ have been introduced for future convenience, following Reisz. A new domain of integration results:

$$
\sigma_{J}=\left\{k^{\prime} \in \mathbf{R}^{4 L} \mid-\frac{\pi}{a}-\Delta_{i \mu}<k_{i \mu}^{\prime} \leq \frac{\pi}{a}-\Delta_{i \mu}\right\}
$$

identical to Reisz's Eq. (4.7) [10].

For the line momenta $\ell_{i}^{B} \in J_{B}, \ell_{j}^{F} \in J_{F}$, (5.7) has the effect

$$
\ell_{i}^{B}(k)=\ell_{i}^{B}\left(k^{\prime}\right)+\frac{2 \pi}{a} z_{i}^{B}, \quad \ell_{j}^{F}(k)=\ell_{j}^{F}\left(k^{\prime}\right)+\frac{\pi}{a} z_{j}^{F}
$$


a generalization of Reisz's (4.5) [10]. When this is accounted for in (5.3), the Heaviside step functions in (5.3) just force $\ell_{i}^{B}\left(k^{\prime}\right) \in J_{B}, \ell_{j}^{F}\left(k^{\prime}\right) \in J_{F}$ into the $\epsilon$-neighborhood of the (unique) pole in $\mathcal{B}_{a}$ and $\mathcal{B}_{2 a}$ respectively. As a consequence the following bounds hold:

$$
\begin{aligned}
& C_{B}^{-1}\left(\ell_{i}^{B} \in J_{B}\right) \leq \alpha_{B}\left(\ell_{i}^{B}\left(k^{\prime}\right)^{2}+\lambda^{2}\right)^{-1}, \\
& C_{F}^{-1}\left(\ell_{j}^{F} \in J_{F}\right) \leq \alpha_{F}\left(\ell_{j}^{F}\left(k^{\prime}\right)^{2}+m^{2}\right)^{-1},
\end{aligned}
$$

generalizations of Reisz's (4.8) [10]. Here, $\alpha_{B}, \alpha_{F}$ are constants that always exist for $\epsilon, a$ sufficiently small. For $\ell_{i} \notin J$, the line momenta are outside of the balls of radius $\epsilon \pi / a$ that are centered on sites of the (reduced) reciprocal lattice for (quarks) gluons. Therefore they are bounded by:

$$
C_{B}^{-1}\left(\ell_{i}^{B} \notin J_{B}\right) \leq \gamma_{B} a^{2}, \quad C_{F}^{-1}\left(\ell_{j}^{F} \notin J_{F}\right) \leq \gamma_{F} a^{2},
$$

generalizations of Reisz's (4.9) [10]. Here, $\gamma_{B}, \gamma_{F}$ are constants that always exist for $\epsilon, a$ sufficiently small.

For the line momenta $\ell_{i}^{B} \notin J_{B}, \ell_{j}^{F} \notin J_{F}$, the shift (15.7) is only guaranteed to have

$$
\ell_{i}^{B}(k)-\ell_{i}^{B}\left(k^{\prime}\right)=C_{i m}^{B} \Delta_{m} \in \frac{\pi}{a} \mathbf{Z}^{4}, \quad \ell_{j}^{F}(k)-\ell_{j}^{F}\left(k^{\prime}\right)=C_{j m}^{F} \Delta_{m} \in \frac{\pi}{a} \mathbf{Z}^{4} .
$$

Whereas $\Theta_{\epsilon}^{F}$ is $\pi / a$-periodic, the function $\Theta_{\epsilon}^{B}$ is only $2 \pi / a$-periodic. Some explicit dependence on $\Delta$ will result, and will be addressed below. Gathering together the various results, we can bound (5.3) by:

$$
\begin{aligned}
\hat{I}_{J z} \leq \alpha_{B}^{h_{B}} & \alpha_{F}^{h_{F}}\left(\gamma_{B} a^{2}\right)^{\left(I_{B}-h_{B}\right)}\left(\gamma_{F} a^{2}\right)^{\left(I_{F}-h_{F}\right)} \\
& \times \int_{\sigma_{J}} d^{4 L} k^{\prime} V\left(k^{\prime}+\Delta, q ; m, a\right) \\
& \times \prod_{i \in J_{B}}\left[\left(\ell_{i}^{B}\left(k^{\prime}\right)^{2}+\lambda^{2}\right)^{-1} \Theta\left(\frac{\pi}{a} \epsilon-\left\|\ell_{i}^{B}\left(k^{\prime}\right)\right\|\right)\right] \\
& \times \prod_{j \in J_{F}}\left[\left(\ell_{j}^{F}\left(k^{\prime}\right)^{2}+m^{2}\right)^{-1} \Theta\left(\frac{\pi}{a} \epsilon-\left\|\ell_{j}^{F}\left(k^{\prime}\right)\right\|\right)\right] \\
& \times \prod_{i \notin J_{B}} \Theta_{\epsilon}^{B}\left(\ell_{i}^{B}\left(k^{\prime}\right)+C_{i m}^{B} \Delta_{m}\right) \prod_{j \notin J_{F}} \Theta_{\epsilon}^{F}\left(\ell_{j}^{F}\left(k^{\prime}\right)\right) .
\end{aligned}
$$

Here, the $h_{B}, h_{F}$ are the number of elements in $J_{B}, J_{F}$ resp.; i.e., the number of line momenta that are $\epsilon$-near to lattice poles. The denominator has been expressed entirely in terms of rational functions. The numerator $V$ requires further study: the shifted loop momentum argument $k^{\prime}+\Delta$ can be accomodated into Reisz's techniques 
to bound the numerator, as will be discussed further in $\$ 5.2$ and $\$ 5.4$ below. It will be seen that the $\Theta, \Theta_{\epsilon}^{B}, \Theta_{\epsilon}^{F}$ functions do not pose any difficulty, as they just restrict the domain of loop integration. With the bound in the form (15.13), it is quite simple to extend the remainder of Reisz's manipulations. Using them, I will formulate and prove the SF power-counting theorem.

\subsection{The numerator}

The magnitude of the SF numerator is also easy to estimate, using the decomposition $J_{F}$ and the shifted line momenta $\ell_{j}\left(k^{\prime}\right)$. However, cancellations associated with the spin-taste algebra will be important to take into account in order to get the correct UV degree for a given diagram. For this reason it is better to abide by Reisz's approach and treat the numerator $V$ as a whole.

I now make a few remarks regarding the effect of the shift $\Delta$ that appears in the numerator of (5.13). This will lead to modifications of propagator numerators and of vertex factors. In the words of Reisz, a generic shift $\Delta_{i} \in \mathbf{R}^{4}$ "would produce explicit negative powers in the lattice spacing destroying convergence." However, though the shift involved here is not an invariance of the Feynman integrand, it is nevertheless special: $\Delta_{i} \in(\pi / a) \mathbf{Z}^{4}$. As I now discuss, it is possible to eliminate this explicit $a^{-1}$ through a transformation in the form of the Feynman integrand. The transformed integrand trades $\sin \leftrightarrow \cos$ in various places, and/or introduces factors of $(-1)$. Furthermore, the number of possibilities for how the propagators and vertex factors are transformed is finite. The UV degree of the transformed numerator is then determined in accordance with Reisz's definition. This degree is then used in a

generalized computation of the UV degree of the integral $\hat{I}$, as will be seen in $\$ 5.3$ below.

As an example of the transformation induced by $\Delta$, consider the quark-gluon vertex (2.13). For the sake of argument, suppose that each line entering the vertex is internal, with

$$
\begin{aligned}
& p \rightarrow \ell_{1}, \quad q \rightarrow \ell_{2}, \quad k \rightarrow \ell_{3}, \\
& \ell_{i}=C_{i j} k_{j}+Q_{i}(q) \quad \forall \quad i=1,2,3 .
\end{aligned}
$$

Thus in the redefinition (5.7) the momentum-dependent factor in the vertex trans- 
forms as:

$$
\begin{aligned}
& e^{i a \ell_{1 \mu}(k)}+e^{-i a\left(\ell_{1 \mu}(k)+\ell_{3 \mu}(k)\right)}= \\
& \quad(-)^{C_{1 i} \delta_{i \mu}}\left[e^{i a \ell_{1 \mu}\left(k^{\prime}\right)}+(-)^{C_{3 i} \delta_{i \mu}} e^{-i a\left(\ell_{1 \mu}\left(k^{\prime}\right)+\ell_{3 \mu}\left(k^{\prime}\right)\right)}\right] .
\end{aligned}
$$

Note that since the line momenta are natural, $C_{i j} \in \mathbf{Z}$. It follows that, as promised, factors of $(-1)$ have been introduced. Momentum conservation implies

$$
C_{1 i}+C_{2 i}+C_{3 i}=0
$$

but this still allows for the transformation (5.15) to have a notrivial effect on the quark-gluon vertex. In the case that $C_{3 i} \delta_{i \mu}=1 \bmod 2$, the factor $\cos \left(\ell_{1 \mu}(k) a+\right.$ $\left.(1 / 2) \ell_{3 \mu}(k) a\right)$ is exchanged for $\sin \left(\ell_{1 \mu}\left(k^{\prime}\right) a+(1 / 2) \ell_{3 \mu}\left(k^{\prime}\right) a\right)$. [Here, it is implicit that overall exponentials are factored out to rewrite the expression using trigonometric functions.] Since the latter starts at $\mathcal{O}(a)$, rather than $\mathcal{O}(1)$, the UV properties of the vertex are changed in a significant way (lowered by 1 ). This does not destroy the convergence of the numerator; in fact, it improves it.

As another example, consider the triple-gluon vertex. In the conventions taken here, ${ }^{9}$

$$
\begin{gathered}
V_{\nu \rho \mu}^{a b c}(p, q, k)=\frac{g}{4} f^{a b c} \delta_{\mu \nu} \bar{\delta}_{a}(p+q+k)\left(e^{i a k_{\rho}}-e^{-i a\left(k_{\rho}+q_{\rho}\right)}\right)\left(1+e^{-i a\left(p_{\mu}+k_{\mu}\right)}\right) \\
+ \text { cyclic permutations. }
\end{gathered}
$$

Applying (5.14), one finds:

$$
\begin{aligned}
& \left(e^{i a \ell_{3 \rho}(k)}-e^{-i a\left(\ell_{3 \rho}(k)+\ell_{2 \rho}(k)\right)}\right)\left(1+e^{-i a\left(\ell_{1 \mu}(k)+\ell_{3 \mu}(k)\right)}\right)= \\
& \quad(-)^{C_{3 i} \delta_{i \rho}}\left(e^{i a \ell_{3 \rho}\left(k^{\prime}\right)}-(-)^{C_{2 i} \delta_{i \rho}} e^{-i a\left(\ell_{3 \rho}\left(k^{\prime}\right)+\ell_{2 \rho}\left(k^{\prime}\right)\right)}\right) \\
& \times\left(1+(-)^{\left(C_{1 i}+C_{2 i}\right) \delta_{i \mu}} e^{-i a\left(\ell_{1 \mu}\left(k^{\prime}\right)+\ell_{3 \mu}\left(k^{\prime}\right)\right)}\right)
\end{aligned}
$$

Here again, the explicit $a^{-1}$ contained in $\Delta_{i}$ is traded for factors of $(-1)$, that in some cases interchange $\sin \leftrightarrow$ cos. Raising or lowering the UV degree by 1 .

An increase in the UV degree of the numerator under transformations such as (5.18) will not "destroy" the "convergence" of the Feynman integral. Rather, it will only make manifest the cutoff dependence. To the extent that a Feynman integral has positive UV degree, subtractions are required in order to have a convergent result, regardless of the basis of loop momenta.

\footnotetext{
${ }^{9}$ See for example Eq. (3.225) of [32], taking into account the $2 \pi / a$-periodic Fourier transform convention (2.4) that I use.
} 
Quite generally, the factors in the numerator $V(k, q ; m, a)$ are trigonometric functions that are $2 \pi / a$-periodic. The redefinition $k_{i}=k_{i}^{\prime}+(\pi / a) \delta_{i}, \delta_{i} \in \mathbf{Z}^{4}$ always results in a half- or full-period shift. The rule is that, prior to computing the UV degree, one should eliminate the explicit $\pi / a$ factor using elementary trigonometric identities, as has just been illustrated for the quark-gluon and triple-gluon vertices.

The best strategy to deal with this is to extend the Feynman rules to incorporate $\pi / a$ shifts. Then the integral (5.13) should be interpreted in terms of these new rules. Symbolically,

$$
\begin{aligned}
& V\left(k^{\prime}+(\pi / a) \delta, q ; m, a\right) \equiv V\left(k^{\prime}+(\pi / a) A, q ; m, a\right) \equiv V_{A}\left(k^{\prime}, q ; m, a\right), \\
& A_{i \mu}=\delta_{i \mu} \quad \bmod 2, \quad A_{i} \in \mathcal{K} .
\end{aligned}
$$

Here, $V_{A}$ is written in terms of the generalized Feynman rules. (Note that in the second step the $2 \pi / a$-periodicity has been used to express the numerator $V$ in terms of the transformed one with an index restricted to $A \in \mathcal{K}^{L}$.) Once this has been done, all of Reisz's techniques for the UV degree analysis of the numerator apply. This can be seen from the fact that the numerator satisfies the Reisz-Lüscher conditions, after the explicit factors of $\pi / a$ have been eliminated. The essential reason for this is that sine and cosine are analytic functions.

\subsection{The generalized theorem}

These considerations lead to the following generalization of Reisz's theorem:

Definition. Let $F_{A}=V_{A} / C_{A}, A \in \mathcal{K}^{L}$ denote the transformed Feynman integrand. That is:

$$
\begin{aligned}
& V_{A}(k, q ; m, a)=V(k+(\pi / a) A, q ; m, a), \\
& C_{A}(k, q ; m, a)=C(k+(\pi / a) A, q ; m, a) .
\end{aligned}
$$

Generalize the UV degree as follows:

$$
\begin{aligned}
& \overline{\overline{\operatorname{degr}}}_{\hat{u}} F=\max _{A \in \mathcal{K}^{L}} \overline{\operatorname{degr}}_{\hat{u}} F_{A}, \quad \overline{\operatorname{degr}}_{\hat{u}} F_{A}=\overline{\operatorname{degr}}_{\hat{u}} V_{A}-\overline{\operatorname{degr}}_{\hat{u}} C_{A} ; \\
& \overline{\overline{\operatorname{degr}}}_{\hat{H}} \hat{I}=4 d+\overline{\overline{\operatorname{degr}}}_{\hat{u}} F .
\end{aligned}
$$

Recall that $u_{1}, \ldots, u_{d}$ parameterizes the Zimmermann subspace $H$.

Proposition. Suppose that 


$$
\overline{\overline{\operatorname{degr}}}_{\hat{H}} \hat{I}<0 \quad \forall \quad H \in \mathcal{H} .
$$

Then $\hat{I}$ converges, and

$$
\lim _{a \rightarrow 0} \hat{I}=\sum_{A \in \mathcal{K}^{L}} \int_{-\infty}^{\infty} d^{4 L} k \frac{P_{A}(k, q ; m)}{E_{A}(k, q ; m)}
$$

where

$$
P_{A}(k, q ; m)=\lim _{a \rightarrow 0} V_{A}(k, q ; m, a), \quad E_{A}(k, q ; m)=\lim _{a \rightarrow 0} C_{A}(k, q ; m, a)
$$

are just the continuum limits of the numerator and denominator resp. This indicates that various regions of loop momenta may contribute to the continuum limit, due to the presence of doublers in the fermion spectrum.

\subsection{Proof}

Starting with (5.3), one makes the redefinition (5.7). Then the numerator is replaced by $V_{A}\left(k^{\prime}, q ; m, a\right)$, as in (5.19). Once this has been done, $\hat{I}_{J z}$ is in the form considered by Reisz. Due to the assumption (5.22), the remainder $R_{A}$ in the decomposition

$$
V_{A}(k, q ; m, a)=P_{A}(k, q ; m)+R_{A}(k, q ; m, a)
$$

does not contribute in the continuum limit, as follows from Reisz's arguments in $\S 7$ of [10]. Thus one can replace $V_{A}$ by the rational function $P_{A}$ in the numerator of $\hat{I}_{J z}$. Furthermore, Reisz's arguments show that the $\hat{I}_{J z}$ term that maps to the index $A \in \mathcal{K}^{L}$ just yields

$$
I_{A}=\int_{-\infty}^{\infty} d^{4 L} k \frac{P_{A}(k, q ; m)}{E_{A}(k, q ; m)}
$$

in the continuum limit. The result (5.23) follows immediately.

To clarify this, I discuss some of the details of $\S 7$ of Reisz [10]. Taking into account the decomposition (5.25) and the effect of (5.7) and (5.19) on (5.3), we have the decomposition

$$
\hat{I}_{J z}=\hat{I}_{J z}^{0}+\hat{I}_{J z}^{R}
$$

where

$$
\begin{aligned}
\hat{I}_{J z}^{0}= & \int_{\sigma_{J}} \frac{d^{4 L} k^{\prime} P_{A}\left(k^{\prime}, q ; m\right)}{C_{A}\left(k^{\prime}, q ; m, a\right)} \\
& \times \prod_{i \in J_{B}} \Theta\left(\frac{\pi}{a} \epsilon-\left\|\ell_{i}^{B}\left(k^{\prime}\right)\right\|\right) \prod_{j \in J_{F}} \Theta\left(\frac{\pi}{a} \epsilon-\left\|\ell_{j}^{F}\left(k^{\prime}\right)\right\|\right) \\
& \times \prod_{i \notin J_{B}} \Theta_{\epsilon}^{B}\left(\ell_{i}^{B}\left(k^{\prime}\right)+C_{i m}^{B} \Delta_{m}\right) \prod_{j \notin J_{F}} \Theta_{\epsilon}^{F}\left(\ell_{j}^{F}\left(k^{\prime}\right)\right),
\end{aligned}
$$


and $\hat{I}_{J z}^{R}$ is defined with $P_{A}$ replaced by $R_{A}$. Note that there is a correspondence between the index $A$ and the index $J z$, since the latter determines the shift (5.7) that is required. When one compares to Reisz's (7.1)-(7.2) of [10], the only difference that one finds is in the factor $\prod_{i \notin J_{B}} \Theta_{\epsilon}^{B}\left(\ell_{i}^{B}\left(k^{\prime}\right)+C_{i m}^{B} \Delta_{m}\right)$, with its extra argument $C_{i m}^{B} \Delta_{m}$. This only restricts the domain of integration; it can only make the integral smaller, so Reisz's bounds still hold. E.g.,

$$
\begin{aligned}
\left|\hat{I}_{J z}^{0}\right| \leq \bar{I}_{J z}^{0}= & \alpha_{B}^{h_{B}} \alpha_{F}^{h_{F}}\left(\gamma_{B} a^{2}\right)^{\left(I_{B}-h_{B}\right)}\left(\gamma_{F} a^{2}\right)^{\left(I_{F}-h_{F}\right)} \\
& \times \int_{\kappa_{J}} \frac{d^{4 L} k^{\prime}\left|P_{A}\left(k^{\prime}, q ; m\right)\right|}{\prod_{i \in J_{B}}\left(\ell_{i}^{B}\left(k^{\prime}\right)^{2}+\lambda^{2}\right) \prod_{j \in J_{F}}\left(\ell_{j}^{F}\left(k^{\prime}\right)^{2}+m^{2}\right)},
\end{aligned}
$$

where $\kappa_{J}$ is the same domain as in Reisz's (7.6) [10]. (Following Reisz, the domain (5.8) is extended, $\sigma_{J} \rightarrow \kappa_{J}$, which can only increase the value of the bounding continuum integral $\bar{I}_{J z}^{0}$.) Similar remarks apply to $\hat{I}_{J z}^{R}$. Note that this is just an exploitation of the bound (5.13).

It follows that the remainder of Reisz's arguments of $\S 7$ [10] apply, with only the following modification. It is still true that $\bar{I}_{J z}^{0}$ has a nonvanishing continuum limit only if all line momenta are near poles: $J_{B}=\left\{1, \ldots, I_{B}\right\}$ and $J_{F}=\left\{1, \ldots, I_{F}\right\}$. However, rather than Reisz's Eq. (7.18) [10], we must write $\left(I=I_{B}+I_{F}\right.$ and $i$ collectively denotes all line momenta):

$$
K_{i}(k)=\sum_{j=1}^{L} C_{i j} k_{j}=\frac{\pi}{a} z_{i}, \quad i=1, \ldots, I,
$$

which differs in that $2 \pi / a$ has been replaced by $\pi / a$ for the coefficient of $z_{i}$. Just as in his case, this equation has a unique solution, due to $\operatorname{rank}\left(C_{i j}\right)=m$. Due to the naturalness of the line momenta, the solution is of the form

$$
k_{i}=(\pi / a) \delta_{i}, \quad \delta_{i} \in \mathbf{Z}^{4} .
$$

These are nothing but the $\delta_{i}$ that are used in the shift (15.7).

Now consider the possible solutions to (5.31), given $k_{i} \in \mathcal{B}_{a}$. Unlike what occurs in the case considered by Reisz, there are multiple possibilities for $\delta_{i}$ that will work. In fact, they are nothing other than the $A_{i} \in \mathcal{K}$. That is, the sum over $A \in \mathcal{K}^{L}$ that is taken in (5.23) is in one-to-one correspondence with the set of $k_{i}=(\pi / a) \delta_{i}$ that are solutions to (5.31). (As will be seen in the example of 95.5 .2 , it can happen that for certain $A \equiv \delta \in \mathcal{K}^{L}$, we get $z_{i} \notin 2 \mathbf{Z}^{4}$ where $i$ corresponds to one of the gluon lines, $\ell_{i}^{B}$. In that case the contribution $I_{A}$ will vanish, since the gluon is always far from the pole in $\bar{I}_{J z}^{0}$.) From this we see that the sum in (5.23) is just the modification that is required to extend the arguments of Reisz's proof. 


\subsection{Simple examples}

\subsubsection{1-loop with external gluons}

Consider any 1-fermion-loop diagram with external gluon legs. ${ }^{10}$ For example, the 4-gluon diagram Fig. 11. Recall that in the MSTB rules, a factor of 1/16 is supplied for each fermion loop, due to the extension of the integration domain. On the other hand, when one takes the continuum limit of the numerator and denominator, only one pole region $(k \approx 0)$ occurs in the continuum Feynman integrand [Eq. (5.26) with $A=(0,0,0,0)$.$] . It is only when we include all 16$ contributions that come from the sum over $A \in \mathcal{K}$ that appears in (5.23) that we get the correct overall factor.

In detail, the denominator of the integrand is invariant under the shift of loop momentum, $k \rightarrow k+(\pi / a) A$, since it involves only the SF propagator. Taking into account the change in the SF propagator numerators and the gluon-quark vertices, it can be shown that the change in the numerator of the integrand is equivalent to just:

$$
\left(\overline{\overline{\gamma_{\mu} \otimes 1}}\right) \rightarrow(-)^{A_{\mu}}\left(\overline{\overline{\gamma_{\mu} \otimes 1}}\right)
$$

in every place that $\left(\overline{\overline{\gamma_{\mu} \otimes 1}}\right)$ appears, and everything else left as it was before the shift in loop momentum. This is just an automorphism of the Clifford algebra, and will not change the value of the character - i.e., the trace that occurs in the numerator. Thus each of the 16 Feynman integrands, $A \in \mathcal{K}$, are identical in their continuum limit to the one obtained at $A=0$. This just cancels the factor of $1 / 16$ to give the desired result.

\subsubsection{1-loop with external fermions}

Next consider diagrams such as Fig. 2. In this case the gluons contribute to the denominator. When the shift $k \rightarrow k+(\pi / a) A$ is performed, with $A \neq 0$, the gluon denominators are moved far from their poles when $k \approx 0$. It follows that $C_{A}^{-1} \sim a^{4}$, where $C_{A}$ is the transformed denominator of the Feynman integrand. The numerator of the integrand $V_{A}$ is well-behaved as $a \rightarrow 0$. Thus for $A \neq 0$ the integrand vanishes in the $a \rightarrow 0$ limit. This is just as required: there is no $1 / 16$ factor to be compensated because we do not have a fermion loop. Only the $A=0$ contribution survives in the $a \rightarrow 0$ limit, and the result is just the usual continuum expression.

\footnotetext{
${ }^{10}$ Vacuum polarization will of course require a subtraction for the power-counting theorem to hold.
} 


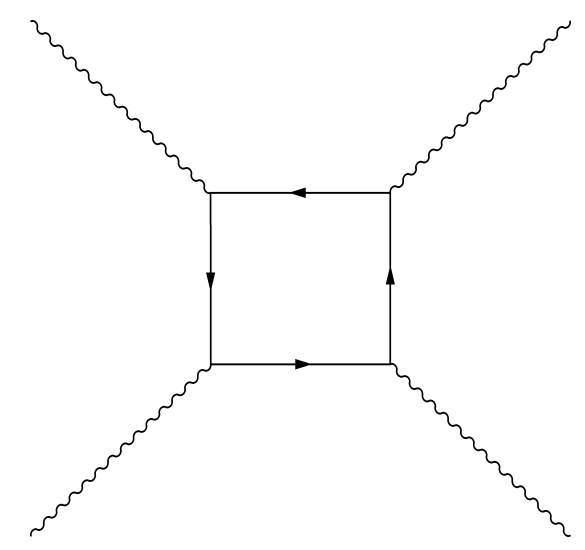

Figure 1: Example of finite diagram where all 16 choices of $A \in \mathcal{K}$ contribute.

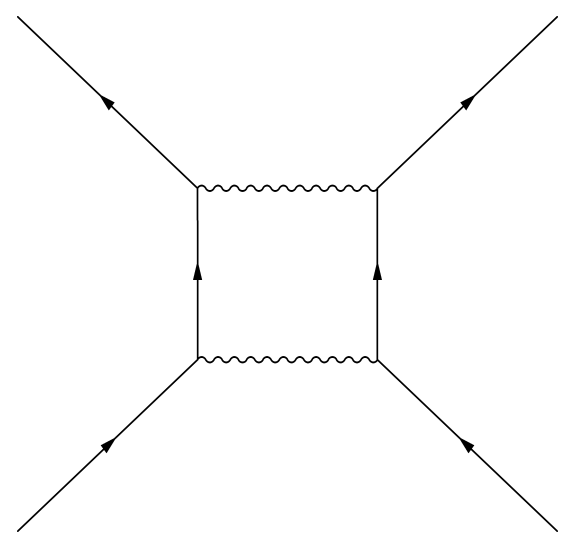

Figure 2: Example of finite diagram where only 1 choice of $A \in \mathcal{K}$ contributes. 


\section{Discussion}

In this article I have demonstrated how to extend the techniques of Reisz's powercounting theorem to the case of staggered fermions. It is fortunate that with a few straightforward modifications, the bulk of Reisz's arguments apply. It is of great practical importance that his power-counting theorem for generalized continuum Feynman integrals ( $\$ 5$ of $[10]$ ) continues to be applicable. One thing that remains to be done is to use the staggered fermion power-counting theorem to prove perturbative renormalizability, following $[7,8]$. Also, applications of the theorem to higher orders in perturbation theory should be explored in more detail. Generalizations to other sorts of theories that contain doublers could also be considered, since the technique that has been introduced here is not very specialized. It is worth emphasizing that all of the manipulations that were performed here apply equally well to improved staggered fermion QCD.

\section{Acknowledgements}

I benefitted from communications with David Adams. This work was supported in part by the U.S. Department of Energy under grant No. DE-FG02-94ER-40823.

\section{Appendices}

\section{A Resolutions of identity}

\section{A.1 Resolution on $\mathcal{B}_{a}$}

In $\S 4$ of [10], Reisz introduces the following step function:

$$
\Theta_{\epsilon}^{B}(\ell)= \begin{cases}0 & \text { if }\left\|\ell-\frac{2 \pi}{a} z\right\|<\frac{\pi}{a} \epsilon \text { for some } z \in \mathbf{Z}^{4} \\ 1 & \text { otherwise. }\end{cases}
$$

A superscript $B$ has been affixed to distinguish it from another step function that will be defined below. For any $\ell$ one can resolve identity as:

$$
1=1_{B}(\ell) \equiv \Theta_{\epsilon}^{B}(\ell)+\sum_{z \in \mathbf{Z}^{4}} \Theta\left(\frac{\pi}{a} \epsilon-\left\|\ell-\frac{2 \pi}{a} z\right\|\right) .
$$

Here, $\Theta$ is Heaviside's unit step function. 


\section{A.2 Resolution on $\mathcal{B}_{2 a}$}

Define a step-function analogous to Reisz's:

$$
\Theta_{\epsilon}^{F}(\ell)= \begin{cases}0 & \text { if }\left\|\ell-\frac{\pi}{a} z\right\|<\frac{\pi}{a} \epsilon \text { for some } z \in \mathbf{Z}^{4}, \\ 1 & \text { otherwise. }\end{cases}
$$

For any $\ell$ one can resolve identity as:

$$
1=1_{F}(\ell) \equiv \Theta_{\epsilon}^{F}(\ell)+\sum_{z \in \mathbf{Z}^{4}} \Theta\left(\frac{\pi}{a} \epsilon-\left\|\ell-\frac{\pi}{a} z\right\|\right) .
$$

As above, $\Theta$ is Heaviside's unit step function. This resolution is useful for line momenta of SFs, since it isolates the regions that are near SF poles.

\section{B Simplification of MSTB rules}

Here I establish a very important simplification that is used to derive the MSTB rules (2.12)-(2.13). It was employed, for instance, by Patel and Sharpe [28].

\section{B.1 $\delta$-function transformation}

Without loss of generality, for any momenta $p, q \in \mathbf{R}^{4}$ we can write

$$
p=p^{\prime}+\frac{\pi}{a} A, \quad q=q^{\prime}+\frac{\pi}{a} B, \quad p^{\prime}, q^{\prime} \in \mathbf{R}^{4}, \quad A, B \in \mathcal{K} .
$$

That is, $p^{\prime}, q^{\prime}$ are not necessarily in the reduced first Brillouin zone $\mathcal{B}_{2 a}$. Even if $p, q$ are integrated over $\mathcal{B}_{a}$, we can impose that $p^{\prime}, q^{\prime}$ are also integrated over $\mathcal{B}_{a}$ by using the equivalence that is established in $\$$ C. In fact, this is exactly what I do when passing to the MSTB from the $1 \mathrm{CB}$. It is also what has been done by Patel and Sharpe [28].

It is not hard to check that the $\delta$-functions that appear in the $1 \mathrm{CB}$ quark propagator (2.8) and vertex (2.9) obey the identities

$$
\begin{aligned}
& \bar{\delta}_{a}\left(p^{\prime}+q^{\prime}+k+\frac{\pi}{a}(A+B+\bar{\mu})\right)= \\
& \prod_{\nu=1}^{4}\left[\bar{\delta}_{a}\left(p_{\nu}^{\prime}+q_{\nu}^{\prime}+k_{\nu}\right) \delta_{A_{\nu}+B_{\nu}+\bar{\mu}_{\nu}, 0}^{[2]}+\bar{\delta}_{a}\left(p_{\nu}^{\prime}+q_{\nu}^{\prime}+k_{\nu}+\frac{\pi}{a}\right) \delta_{A_{\nu}+B_{\nu}+\bar{\mu}_{\nu}, 1}^{[2]}\right],
\end{aligned}
$$


with $k=0$ in the quark propagator (2.8), and $\delta^{[2]}$ a Krönecker $\delta \bmod 2$. It is worth noting that in the transition from (2.8)-(2.9) to (2.12)-(2.13), the identity ${ }^{11}$

$$
\left(\overline{\overline{\gamma_{\mu} \otimes 1}}\right)_{A, B}=(-)^{A_{\mu}} \prod_{\nu=1}^{4} \delta_{A_{\nu}+B_{\nu}+\bar{\mu}_{\nu}, 0}^{[2]} \equiv(-)^{A_{\mu}} \delta_{A+B+\bar{\mu}, 0}^{[2]}
$$

is used. The factor $(-)^{A_{\mu}}$ is obtained from the $p$-dependent prefactors of the $\bar{\delta}_{a^{-}}$ functions in (2.8)-(2.9) under $p=p^{\prime}+(\pi / a) A$. However, the other $\delta$-functions that occur in (B.2) need to be taken into account. Next I will demonstrate the equivalence that allows us to eliminate the $\delta$ functions that violate momentum conservation by $\pi / a$. In the proof the power-counting theorem, this is key to obtaining natural line momenta in the continuum, bounding integrals $\bar{I}_{J z}^{0}$ and $\bar{I}_{J z}^{R}$.

\section{B.2 Equivalence}

As mentioned above, the trick of $₫ \mathbb{C}$ is used to extend the integration of $p^{\prime}, q^{\prime}$ to $\mathcal{B}_{a}$. Then a typical integral against the $\bar{\delta}_{a}(\cdots+\pi / a)$ parts of (B.2) takes the form:

$$
\begin{aligned}
I= & \frac{1}{(16)^{2}} \int_{-\pi / a}^{\pi / a} d p_{\nu}^{\prime} \int_{-\pi / a}^{\pi / a} d q_{\nu}^{\prime} \int_{-\pi / a}^{\pi / a} d k_{\nu} \sum_{A, B} \\
& \times \bar{\delta}_{a}\left(p_{\nu}^{\prime}+q_{\nu}^{\prime}+k_{\nu}+\frac{\pi}{a}\right) \delta_{A_{\nu}+B_{\nu}+\bar{\mu}_{\nu}, 1}^{[2]} f\left(p_{\nu}^{\prime}+\frac{\pi}{a} A_{\nu}, q_{\nu}^{\prime}+\frac{\pi}{a} B_{\nu}, k_{\nu} ; \ldots\right)
\end{aligned}
$$

where $f$ represents the rest of the integrand, where "..." corresponds to integrals over other momenta components and other momenta. I hide integrations over these other variables, for simplicity of notation. The functional form of $f$, namely the way that $p^{\prime}$ and $A$ appear together, etc., is guaranteed by the fact that we start from the $1 \mathrm{CB}$.

Next I make the redefinitions

$$
q_{\nu}^{\prime} \rightarrow q_{\nu}^{\prime}-\frac{\pi}{a}, \quad B_{\nu} \rightarrow B_{\nu}+1
$$

to obtain

$$
\begin{aligned}
I= & \frac{1}{(16)^{2}} \int_{-\pi / a}^{\pi / a} d p_{\nu}^{\prime} \int_{0}^{2 \pi / a} d q_{\nu}^{\prime} \int_{-\pi / a}^{\pi / a} d k_{\nu} \sum_{A, B} \\
& \times \bar{\delta}_{a}\left(p_{\nu}^{\prime}+q_{\nu}^{\prime}+k_{\nu}\right) \delta_{A_{\nu}+B_{\nu}+\bar{\mu}_{\nu}, 0}^{[2]} f\left(p_{\nu}^{\prime}+\frac{\pi}{a} A_{\nu}, q_{\nu}^{\prime}+\frac{\pi}{a} B_{\nu}, k_{\nu} ; \ldots\right) .
\end{aligned}
$$

\footnotetext{
${ }^{11}$ This identity is easily checked using, for example, the definitions given in $\S 2.1-2.2$ of [28].
} 
Finally, since the integration over $q_{\nu}^{\prime}$ is a full period, we can shift the domain

$$
\int_{0}^{2 \pi / a} d q_{\nu}^{\prime} \rightarrow \int_{-\pi / a}^{\pi / a} d q_{\nu}^{\prime}
$$

at no cost. This establishes the equivalence $\bar{\delta}_{a}(\cdots+\pi / a)$ to the momentum conserving terms $\bar{\delta}_{a}(\cdots)$. In essence, we trade momentum violation by $\pi / a$ for a shift in taste. Thus we find that under the integration $\int_{\mathcal{B}_{a}} d^{4} q^{\prime}$,

$$
\begin{aligned}
& \prod_{\nu=1}^{4}\left[\bar{\delta}_{a}\left(p_{\nu}^{\prime}+q_{\nu}^{\prime}+k_{\nu}\right) \delta_{A_{\nu}+B_{\nu}+\bar{\mu}_{\nu}, 0}^{[2]}+\bar{\delta}_{a}\left(p_{\nu}^{\prime}+q_{\nu}^{\prime}+k_{\nu}+\frac{\pi}{a}\right) \delta_{A_{\nu}+B_{\nu}+\bar{\mu}_{\nu}, 1}^{[2]}\right] \\
& \simeq 16 \bar{\delta}_{a}\left(p^{\prime}+q^{\prime}+k\right) \delta_{A+B+\bar{\mu}, 0}^{[2]} .
\end{aligned}
$$

We could also have used the $p^{\prime}$ integration in these manipulations. If 2 momenta are external, we cannot use this equivalence and more care is required [33]. This technicality does not affect the power-counting considerations here, because we are only interested in 1PI loop diagrams (the aim is to study renormalization), which do not contain vertices with 2 external momenta.

\section{Domain extension in MSTB}

Here I prove that after the decomposition (B.1) of momenta for the transition $1 \mathrm{CB}$ $\rightarrow$ MSTB, we can double the domain of integration for $p^{\prime}$, etc. The point is to obtain integration over a full period. It also is invoked in the simplification of $\$ \mathrm{~B}$.

In the manipulations of this paragraph, $p$ will denote any of the components $p_{\mu}$ of a momentum that is integrated over in the Feynman rules in the $1 \mathrm{CB}$. We use equivalence of integration over any full period to write:

$$
\int_{-\pi / a}^{\pi / a} d p \simeq \int_{-\pi / 2 a}^{3 \pi / 2 a} d p=\frac{1}{2}\left(\int_{-\pi / 2 a}^{3 \pi / 2 a} d p_{1}+\int_{-\pi / 2 a}^{3 \pi / 2 a} d p_{2}\right)
$$

where in the last step the integration has been prepared for further manipulations. Then we decompose $p_{1}, p_{2}$ onto separate reduced domains:

$$
\begin{aligned}
& p_{1}=p_{1}^{\prime}+\frac{\pi}{a} A_{1}, \quad p_{1}^{\prime} \in(-\pi / 2 a, \pi / 2 a], \quad A_{1} \in\{0,1\}, \\
& p_{2}=p_{2}^{\prime}-\frac{\pi}{a} A_{2}, \quad p_{2}^{\prime} \in(\pi / 2 a, 3 \pi / 2 a], \quad A_{2} \in\{0,1\} .
\end{aligned}
$$


The integration of (C.1) can then be expressed equivalently as:

$$
\frac{1}{2}\left(\int_{-\pi / 2 a}^{\pi / 2 a} d p_{1}^{\prime} \sum_{A_{1}}+\int_{\pi / 2 a}^{3 \pi / 2 a} d p_{2}^{\prime} \sum_{A_{2}}\right)=\frac{1}{2} \sum_{A} \int_{-\pi / 2 a}^{3 \pi / 2 a} \simeq \frac{1}{2} \sum_{A} \int_{-\pi / a}^{\pi / a},
$$

where in the last step I have again used the equivalence of integrations over a full period.

Extending this manipulation to all components, we obtain the identity

$$
\int_{\mathcal{B}_{a}} d^{4} p(\cdots)=\frac{1}{16} \sum_{A \in \mathcal{K}} \int_{\mathcal{B}_{a}} d^{4} p^{\prime}(\cdots)_{p \equiv p^{\prime}+\frac{\pi}{a} A}
$$

\section{References}

[1] J. B. Kogut and L. Susskind, "Hamiltonian Formulation Of Wilson's Lattice Gauge Theories," Phys. Rev. D 11, 395 (1975).

[2] L. Susskind, "Lattice Fermions," Phys. Rev. D 16, 3031 (1977).

[3] H. S. Sharatchandra, H. J. Thun and P. Weisz, "Susskind Fermions On A Euclidean Lattice," Nucl. Phys. B 192 (1981) 205.

[4] C. T. H. Davies et al. [HPQCD Collaboration], "High-precision lattice QCD confronts experiment," Phys. Rev. Lett. 92, 022001 (2004) arXiv:hep-lat/0304004.

[5] C. DeTar, S. Gottleib, "Lattice Quantum Chromodynamics Comes of Age," Physics Today, February (2004) 45.

[6] M. Lüscher, "Selected Topics In Lattice Field Theory," in "Fields, Strings and Critical Phenomena," Les Houches XLIX, 1988, eds. E. Brézin and J. ZinnJustin, Elsevier, New York, 1989.

[7] T. Reisz, "Lattice Gauge Theory: Renormalization To All Orders In The Loop Expansion," Nucl. Phys. B 318 (1989) 417.

[8] T. Reisz, "Renormalization Of Feynman Integrals On The Lattice," Commun. Math. Phys. 117 (1988) 79.

[9] T. Reisz, "Renormalization Of Lattice Feynman Integrals With Massless Propagators," Commun. Math. Phys. 117 (1988) 639. 
[10] T. Reisz, "A Power Counting Theorem For Feynman Integrals On The Lattice," Commun. Math. Phys. 116 (1988) 81.

[11] T. Reisz, "A Convergence Theorem For Lattice Feynman Integrals With Massless Propagators," Commun. Math. Phys. 116 (1988) 573.

[12] T. Reisz, "Power Counting And Renormalization In Lattice Field Theory," in Proceeding, Nonperturbative Quantum Field Theory, Cargese, 1987.

[13] T. Reisz, "Renormalization Theory On The Lattice With Application To Lattice Gauge Theory," MPI-PAE/PTh-79/88, invited talk given at S.C.R.I., Florida State U., Tallahassee, Oct 1988, and invited talk given at Pisa Univ., Pisa, Italy, Dec 1988.

[14] J. Giedt, R. Koniuk, E. Poppitz and T. Yavin, "Less naive about supersymmetric lattice quantum mechanics," JHEP 0412 (2004) 033 arXiv:hep-lat/0410041.

[15] K. Jansen, "Actions for dynamical fermion simulations: Are we ready to go?," Nucl. Phys. Proc. Suppl. 129, 3 (2004) arXiv:hep-lat/0311039.

[16] M. Pernici, M. Raciti and F. Riva, "Renormalization of matter field theories on the lattice and the flow equation," Nucl. Phys. B 458 (1996) 355 arXiv:hep-th/9507055.

[17] D. H. Adams, "Testing universality and the fractional power prescription for the staggered fermion determinant," Nucl. Phys. Proc. Suppl. 140 (2005) 148 arXiv:hep-lat/0409013.

[18] L. Lellouch, "Flavour physics from lattice QCD," proceedings Continuous advances in QCD, ed. M. Peloso, FTPI, U. Minnesota, Minneapolis, May 11-14, 2006.

[19] M. F. L. Golterman and J. Smit, "Self-energy and flavor interpretation of staggered fermions," Nucl. Phys. B 245 (1984) 61.

[20] S. Weinberg, "High-Energy Behavior In Quantum Field Theory," Phys. Rev. 118 (1960) 838 .

[21] Y. Hahn, W. Zimmermann, "An elementary proof of Dyson's power counting theorem," Commun. Math. Phys., 10 (1968) 330. 
[22] J. M. Rabin, "Homology Theory Of Lattice Fermion Doubling," Nucl. Phys. B 201 (1982) 315.

[23] T. Banks, Y. Dothan and D. Horn, "Geometric Fermions," Phys. Lett. B 117 (1982) 413.

[24] P. Becher, "Dirac Fermions On The Lattice: A Local Approach Without Spectrum Degeneracy," Phys. Lett. B 104 (1981) 221.

[25] P. Becher and H. Joos, "The Dirac-Kahler Equation And Fermions On The Lattice," Z. Phys. C 15 (1982) 343.

[26] P. Becher and H. Joos, "On The Geometric Lattice Approximation To A Realistic Model Of QCD," Lett. Nuovo Cim. 38 (1983) 293.

[27] D. Daniel and S. N. Sheard, "Perturbative Corrections To Staggered Fermion Lattice Operators," Nucl. Phys. B 302 (1988) 471.

[28] A. Patel and S. R. Sharpe, "Perturbative corrections for staggered fermion bilinears," Nucl. Phys. B 395, 701 (1993) arXiv:hep-lat/9210039.

[29] F. Gliozzi, "Spinor Algebra Of The One Component Lattice Fermions," Nucl. Phys. B 204 (1982) 419.

[30] H. Kluberg-Stern, A. Morel, O. Napoly and B. Petersson, "Flavors Of Lagrangian Susskind Fermions," Nucl. Phys. B 220 (1983) 447.

[31] H. J. Rothe, "Lattice gauge theories: An Introduction," World Sci. Lect. Notes Phys. 74 (2005) 1.

[32] I. Montvay and G. Munster, "Quantum Fields on a Lattice," (Cambridge University Press, 1994).

[33] S. R. Sharpe and A. Patel, "Perturbative corrections for staggered four fermion operators," Nucl. Phys. B 417 (1994) 307 arXiv:hep-lat/9310004. 